\title{
El paisaje rural antropogénico de Aisén, Chile: Estructura y dinámica de la vegetación
}

\section{The anthropogenic rural landscape of Aisén, Chile: Structure and vegetation dynamic}

\author{
Carlos Ramírez ${ }^{1 *}$, Víctor Sandoval ${ }^{2}$, Cristina San Martín ${ }^{3}$, Miguel Álvarez $^{4}$, Yéssica Pérez ${ }^{3}$ \& \\ CARLA NOVOA ${ }^{5}$
}

\begin{abstract}
${ }^{1}$ Departamento de Ecología, Pontificia Universidad Católica de Chile, Alameda №340, Santiago, Chile. ${ }^{2}$ Instituto de Geomática, Universidad Austral de Chile, Casilla 567, Valdivia, Chile. ${ }^{3}$ Instituto de Ciencias Ambientales y Evolutivas, Universidad Austral de Chile, Casilla 567, Valdivia, Chile. ${ }^{4}$ INRES-Vegetationsökologie, Rheinische Friedrich-Wilhelms-Universität, Bonn, Alemania. ${ }^{5}$ Institut für Geobotanik, Leibnitz Universität Hannover, Nienburger Strasse 17, D-30167, Alemania. *cramirez@uach.cl
\end{abstract}

\begin{abstract}
RESUMEN
Se estudió la dinámica de degradación de la vegetación en un mosaico vegetal antropogénico del paisaje rural de la Región de Aisén para establecer las posibles relaciones de origen tanto espacial como temporal entre las comunidades y proponer series de degradación. Se trabajó con metodología fitosociológica levantando 50 censos de vegetación. La tabla inicial fue ordenada usando especies diferenciales en 7 comunidades: matorral de ñire (Anemone multifida-Nothofagus antarctica), silvopastoreo con ñire, silvopastoreo con chacay, pradera de trébol-ballica (Bromus catharticus-Trifolium repens), pradera de chépica-cadillo (Acaena ovalifolia-Agrostis capillaris), pradera de cadillo-Aira y estepa de cadillo, las que se compararon en sus espectros sistemáticos, biológicos y de origen. Posteriormente, se realizaron análisis estadísticos multivariados de clasificación (conglomerados) y de ordenación (componentes principales) a la tabla inicial transformada previamente en una matriz de datos. Finalmente, utilizando el índice de Ellenberg se compararon las comunidades encontradas, las que se distribuyeron en una constelación de comunidades. La flora de la tabla inicial estuvo conformada por 78 especies con un predominio de plantas herbáceas perennes y anuales alóctonas que indican un alto grado de antropización. Se estableció la siguiente dinámica vegetacional de degradación: el matorral de ñire se degrada por pastoreo a un silvopastoreo con ñire, el cual se transforma en una pradera antropogénica, con sobrepastoreo ésta da origen a una pradera de cadillo-Aira, la que por abandono desemboca en una estepa de cadillo. Se discuten los resultados obtenidos y la aplicabilidad del método.
\end{abstract}

Palabras clave: Patagonia chilena, vegetación, dinámica, degradación antrópica.

\begin{abstract}
We studied the dynamic of the vegetation degradation in a rural anthropogenic mosaic vegetal landscape of the Aisen Region, Chile to establish relations of origin in space and time between communities and propose series of degradation. With plant sociological methodology we make up 50 vegetation samples. The initial table was sorted using differential species in 7 communities: ñire scrub (Anemone multifida-Nothofagus antarctica), silvipasture with ñire, silvipasture with chacay, trebol-ballica prairie (Bromus catharticus-Trifolium repens), chepica-cadillo prairie (Acaena ovalifolia-Agrostis capillaris), cadillo-Aira prairie and cadillo steppe, which were compared in their systematic, biological and origin spectra. The initial table previously transformed into a data matrix was treated with multivariate statistical analysis of classification (cluster analysis) and ordination (principal components analysis). Finally using the Ellenberg community similarity coefficient were compared and distributed in a communities constellation. The flora of the initial plant sociological table consisted of 78 species with a predominance of annual and perennial herbaceous foreign species that indicated a large anthropic influence. The following vegetation dynamic of degradation was established: ñire scrub is degraded by grazing in a ñire silvipasture, which is transformed in a anthropogenic overgrazed trebol-ballica prairie this becomes a cadillo-Aira prairie, which by default ends in a cadillo steppe without human management possibility. We discussed the results and applicability of the using methods.
\end{abstract}

KeYwords: Chilean Patagonia, vegetation, dynamic, anthropic degradation. 


\section{INTRODUCCIÓN}

El mosaico de vegetación que conforma el paisaje vegetal rural antropogénico representa generalmente, una situación seminatural en la cual conviven rodales de las comunidades vegetales originales (primarias) con aquéllas de reemplazo (secundarias y terciarias) productos de la intervención humana (Ramírez et al. 2003, Aguayo et al. 2009, San Martín et al. 2009). De manera que en todo paisaje vegetal antropizado existió una situación primitiva original, sin intervención humana (vegetación potencial) que para la región estudiada ha sido caracterizada por Luebert \& Pliscoff (2006), la que alterada por el ser humano con sus manejos silvoagropecuarios posibilita la instalación de nuevas comunidades vegetales que conviven con los restos de las primitivas (vegetación real o actual) (Terradas 2006).

La dominancia de especies nativas se presenta en comunidades primarias poco alteradas, un equilibrio entre ambos orígenes (nativo e introducidas) aparece en comunidades secundarias de reemplazo y la dominancia de especies introducidas indica comunidades terciarias que se forman sobre comunidades secundarias pratenses muy alteradas por el manejo, donde el grado de antropización o hemerobia es muy alto (Frey \& Lösch 2010). En las comunidades terciarias las condiciones ambientales se vuelven extremas, por lo que en ellas no pueden sobrevivir malezas adaptadas a condiciones de cultivo y pastoreo, por esta razón vuelven a figurar especies nativas, que al no tener competencia colonizan el lugar.

Aunque la degradación antropogénica de la vegetación primaria humaniza el paisaje, la situación real es que con un mayor tiempo de uso, los suelos se degradan y se vuelven improductivos (Ellies et al. 1994). En procesos naturales de destrucción o alteración de la vegetación primitiva (sequía, inundación, derrumbes, etc.), al volverse a la situación normal, la vegetación potencial puede recuperarse, siempre que no haya intervención antrópica (Rosas 1991).

Comprender la dinámica de estos procesos degradativos que conforman la vegetación real es muy importante, ya que por un lado permiten establecer qué manejo provoca determinada alteración $\mathrm{y}$, por otro, inferir cuál pudo ser la situación original (vegetación potencial), lo que le da carácter deductivo y predictivo a este tipo de estudios (Ramírez et al. 2003). Esto puede servir de base para la planificación y manejo del paisaje, el uso del suelo y la restauración de ambos.

Las preguntas recurrentes en este tipo de investigaciones son ¿cuál es el origen de cada una de las comunidades vegetales que integran el paisaje? y ¿cuál es la relación entre ellas? Responder a estas preguntas no es fácil, ya que no siempre la proximidad espacial indica estas relaciones y la proximidad temporal frecuentemente se desconoce, además, algunas comunidades pueden tener un origen mixto, presentando rodales originales (primarios) y otros, productos de la degradación del suelo provocada por el manejo y el posterior abandono (secundarios y terciarios). Por ello y para facilitar este tipo de investigaciones, se propone agregar, a la proximidad espacial y temporal, la proximidad florística con métodos estadísticos multivariados de clasificación y de ordenación (Ramírez et al. 1984), el origen fitogeográfico y las formas de vida y, con ellos, ubicar el lugar de cada comunidad en la serie de sucesión secundaria de degradación de la vegetación original.

El presente estudio pone a prueba estas proposiciones metodológicas en un paisaje antropizado de la comuna de Cochrane en la provincia Capitán Prat de la Región de Aisén, Chile, teniendo como objetivos la determinación de las comunidades vegetales que lo integran y, principalmente, establecer el curso seguido por la dinámica de degradación antropogénica de la vegetación leñosa primaria.

\section{MATERIALES Y MÉTODOS}

\section{LUGAR DE TRABAJO}

El lugar estudiado corresponde a un cordón montañoso ubicado a unos $10 \mathrm{~km}$ al norte de la localidad de Cochrane y al noreste del río Baker, entre la Balsa Baker y la pasarela del sector El Manzano, a una altitud promedio de $459 \mathrm{~m}$ snm (mínima 360 y máxima $514 \mathrm{~m} \mathrm{snm}$ ) recorrido por un camino vecinal que une ambos lugares donde se cruza el río (Fig. 1).

La formación vegetal original dominante es el matorral de Nothofagus antarctica (G.Forst.) Oerst. (ñire), en algunos sectores protegidos aparece bosque de Nothofagus pumilio (Poeep et Endl.) Krasser (lenga), en lugares rocosos y secos de la ladera que mira al río Baker prosperan bosques de Nothofagus obliqua (Mirb.) Oerst. (coihue) y matorrales de Colletia hystrix Clos (yaqui) (Vidal et al. 2011, Amigo \& Rodríguez-Guitián 2011). Esta vegetación primaria se encuentra muy alterada, principalmente por pastoreo y extracción de leña y madera (Reque et al. 2007), en el pasado también el fuego fue un agente importante en la destrucción de los bosques (Veblen et al. 1995), de manera que en la actualidad hay un mosaico de comunidades arbustivas, pratenses y esteparias.

El clima es frío del tipo estepario de altura con grandes variaciones térmicas entre invierno y verano, en el primero hay precipitación nival y en el segundo bastante calor $\mathrm{y}$ sequía. La estación meteorológica más cercana corresponde a Chile Chico, ubicado en la estepa patagónica a orillas del lago General Carrera y cerca del límite con Argentina, en el diagrama climático de este lugar se observa la influencia continental con marcadas diferencias estacionales de precipitación y temperatura (Fig. 2). La pluviosidad promedio anual es de $191 \mathrm{~mm}$ y la temperatura promedio anual de $11,5^{\circ} \mathrm{C}$, las temperaturas promedio mínima $\mathrm{y}$ máxima alcanzan a $5,1^{\circ} \mathrm{C}$ y a $15,4^{\circ} \mathrm{C}$, respectivamente (Hajek \& Di Castri 1975). Esta estación meteorológica ha sido clasificada en el cinturón bioclimático supratemperado y en el ombrotipo seco por Amigo \& Ramírez (1998). 

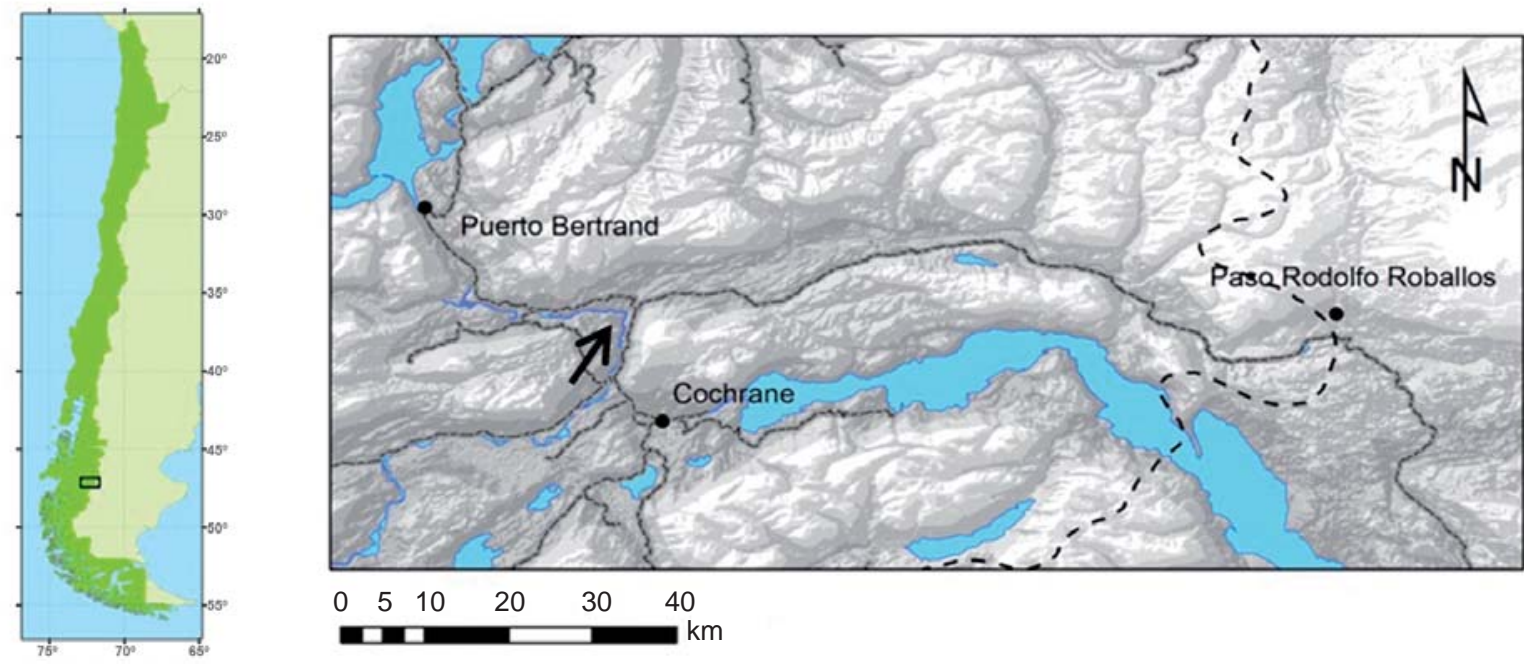

Figura 1. Ubicación del lugar de trabajo (flecha negra) en la Región de Aisén, Chile.

Figure 1. Location of the work place (black arrow) in the Aisen Region, Chile.

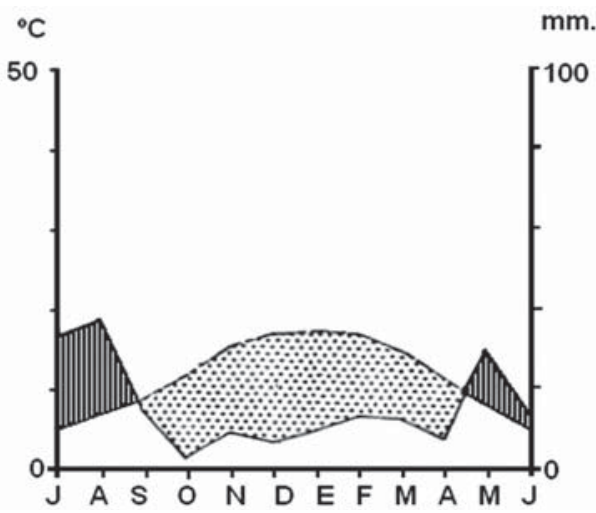

Figura 2. Diagrama climático de Chile Chico (46ㅇ $36^{\prime} \mathrm{S} ; 71^{\circ} 43^{\prime} \mathrm{W}$; Altitud 382 m) según Hajek \& Di Castri (1975).

Figure 2. Clima diagram from Chile Chico $\left(46^{\circ} 36^{\prime} \mathrm{S} ; 71^{\circ} 43^{\prime} \mathrm{W}\right.$; Altitude $382 \mathrm{~m}$ ) after Hajek \& Di Castri (1975).

\section{MÉTodos}

En el lugar de trabajo se diferenciaron las unidades de vegetación existentes en el paisaje (formaciones vegetales) $\mathrm{y}$ en sectores florística-, fisonómica- y ecológicamente homogéneos de ellas se procedió a levantar censos de vegetación con la metodología fitosociológica (Kreeb 1983). En cada parcela de muestreo, cuyo tamaño se correspondía con el tipo de formación a la que pertenecía superando siempre el área mínima correspondiente, de $25 \mathrm{~m}^{2}$ para praderas, $100 \mathrm{~m}^{2}$ para matorrales y estepas y de $400 \mathrm{~m}^{2}$ para bosques (Knapp 1984), se anotaron las especies vegetales y luego se estimó la abundancia de cada una de ellas, mediante la cobertura expresada en porcentaje (Ramírez et al. 1997). Valores inferiores a $1 \%$ se subieron a la unidad. Si la parcela presenta estratificación, la suma de las coberturas de las especies de cada censo puede superar el 100\%.

Se levantaron 50 censos de vegetación, ellos fueron reunidos en una tabla inicial que en su primera columna lleva las especies y en las restantes, la cobertura (expresada en porcentaje) para cada uno en los censos (Anexo 1). De la primera columna se obtuvo la lista florística del lugar, la que fue determinada y clasificada de acuerdo a Moore (1983), Matthei (1995) y Rodríguez et al. (2008), Se determinaron además, el origen fitogeográfico (nativo e introducido) y formas de vida: fanerófitos (plantas leñosas), caméfitos (subarbustos, plantas pulvinadas y hierbas altas), hemicriptófitos (hierbas perennes), criptófitos (geófitos, helófitos e hidrófitos) y terófitos (hierbas anuales y bianuales) para cada especie (Mueller-Dombois \& Ellenberg 1974), construyendo con los resultados los correspondientes espectros taxonómicos, de origen y biológico (Cain 1950). Para ordenar las especies de la tabla de vegetación y conocer su relevancia en ella, se calculó un valor de importancia de acuerdo a Wikum \& Schanholtzer (1978) que, considerando tanto la frecuencia como la cobertura, carece de unidad de medida y tiene un valor máximo posible de 200.

La tabla de vegetación inicial que reunía los 50 censos fue ordenada de forma tradicional para distinguir comunidades, utilizando especies diferenciales que se excluyen mutuamente, este proceso sirvió para separar y determinar las comunidades vegetales presentes en el lugar (Braun-Blanquet 1979) que fueron nominadas de acuerdo a las normas actuales de nomenclatura vegetacional (Izco \& Del Arco 2003). Posteriormente, la tabla inicial, transformada en una matriz de datos homogeneizada en su varianza (abundancia $+1 \log$ ), fue sometida a análisis estadísticos multivariados de clasificación (análisis de 
conglomerados UPGMA) y de ordenación (análisis de componentes) utilizando la distancia Euclidiana como medida de similitud. Se trabajó con el paquete estadístico PCOrd (McCune \& James 2002, Leyer \& Wesche 2007).

En una tabla resumida, con todas las especies en las filas, pero sólo con las comunidades encontradas en las columnas, y con valores promedios de cobertura se aplicó el índice de comunidad de Ellenberg (Frey \& Lösch 2010), formando con los resultados dos matrices simétricas: una de similitud y la otra de disimilitud, que fueron utilizadas como base para diseñar una constelación de comunidades que muestra la cercanía florística (composición florística y abundancia) entre ellas (De Vries et al. 1954, Dierschke 1994). Además, se cálculo la heterogeneidad de las comunidades utilizando el índice de Bray-Curtis (Bray \& Curtis 1957).

\section{Conceptos UTILIZAdos}

A falta de otro concepto en idioma español se utilizará la palabra "silvopastoreo" para designar el pastoreo realizado en rodales boscosos o de matorral cuyo sotobosque original ha sido retirado artificialmente o destruido por el pisoteo y ramoneo del ganado y reemplazado por una cubierta herbácea. Se utilizarán además, los nombres comunes de las comunidades estudiadas ya que sólo algunas han sido formalmente descritas (ver Tabla III) y otras corresponden a estadios intermedios de degradación del bosque o matorral para el establecimiento de praderas permanentes. Por la misma razón, los grupos de rodales estudiados serán tratados como "comunidades", que en fitosociología corresponden a sintaxa de rango desconocido.

\section{RESULTADOS}

La tabla inicial construida con 50 censos contiene 78 especies, de ellas, 49 (62,83\%) son nativas y 29 (37,17\%) introducidas, sin embargo esta proporción cambia drásticamente al considerar la contribución de estos grupos a la cobertura total, en este caso las plantas nativas sólo contribuyen con $1.623(31,29 \%)$ y las introducidas con 3.592 (68,71\%). Esta dominancia de especies introducidas en cuanto a cobertura (abundancia) indica una fuerte degradación antrópica del paisaje vegetal original (Tabla I). Lo anterior se refuerza al considerar el valor de importancia ya que los primeros y más altos valores corresponden a Taraxacum officinale $(25,61)$ y Agrostis capillaris (18,21), el tercer lugar lo ocupa la especie nativa Acaena integerrima (16,98), pero luego se presentan nuevamente tres especies introducidas, Holcus lanatus $(13,45)$, Achillea millefolium $(11,45)$ y Trifolium repens $(10,15)$.

Ninguna especie estuvo presente en los 50 censos de la tabla inicial, la que mayor frecuencia presentó fue Holcus lanatus, especie alóctona que se detectó en 43 censos, esto indica una alta heterogeneidad en la vegetación, lo que se refleja en la presencia de varias comunidades vegetales. Después de esta especie y en orden descendente, se disponen 6 introducidas: Taraxacum officinale, Achillea millefolium, Poa pratensis, Rumex acetosella, Cerastium arvense y Trifolium repens. Recién en noveno y décimo lugar figuran dos especies nativas Acaena integerrima y Nothofagus antarctica, respectivamente. Esta alta frecuencia de especies alóctonas confirma, una vez más, el alto grado de antropización en que se encuentra el paisaje en la región estudiada.

TABLA I. Origen y formas de vida de la totalidad de la flora presente en la tabla inicial, por especies y cobertura.

TABLE I. Origin and life forms of the total flora from initial vegetation table, by species and by cover.

\begin{tabular}{|c|c|c|c|c|}
\hline & \multicolumn{2}{|c|}{ ESPECIES } & \multicolumn{2}{|c|}{ Cobertura } \\
\hline & NÚMERO & Porcentaje & COBERTURA TOTAL & Porcentaje \\
\hline \multicolumn{5}{|l|}{ ORIGEN } \\
\hline Nativas & 49 & 62,82 & 1632 & 31,29 \\
\hline Introducidas & 29 & 37,18 & 3583 & 68,71 \\
\hline Total & 78 & 100 & 5215 & 100 \\
\hline \multicolumn{5}{|l|}{ FORMAS DE VIDA } \\
\hline Fanerófitos & 16 & 20,51 & 614 & 11,77 \\
\hline Caméfitos & 8 & 10,26 & 798 & 15,30 \\
\hline Hemicriptófitos & 36 & 46,15 & 3260 & 62,51 \\
\hline Criptófitos & 3 & 3,85 & 4 & 0.07 \\
\hline Terófitos & 14 & 17,95 & 539 & 10,33 \\
\hline Musgos & 1 & 1,28 & 1 & 0.02 \\
\hline Total & 78 & 100 & 5215 & 100 \\
\hline
\end{tabular}


En las especies determinadas dominan las Dicotiledóneas (incluidas Angiospermas basales) con 59 especies y 75,64\%; representadas con menor cantidad figuran las Monocotiledóneas con 17 especies y 21,79\%. Musgos y Helechos son más escasos.

La forma de vida más abundante, hemicriptófitos estuvo representada por 36 especies, lo que corresponde a un $46,15 \%$ del total, señalando con ello una importante alteración antrópica (Tabla I). Aunque originalmente el lugar estudiado correspondía a vegetación arbustiva o boscosa, las plantas leñosas (fanerófitos) presentan sólo 16 especies $(20,51 \%)$ del total de 78 especies vegetales. Siguen las hierbas anuales (terófitos) con 14 (17,95\%) especies, la abundante presencia de éstas también se relaciona con el alto grado de antropización del lugar. Los caméfitos subarbustivos, propios de vegetación esteparia, presentan $8(10,26 \%)$ especies. Las otras dos formas de vida están escasamente representadas. Al considerar la cobertura total (y porcentaje) de cada forma de vida, el orden de abundancia se mantiene, pero hemicriptófitos y caméfitos aumentan considerablemente. Por el contrario las formas de vida fanerófitos, terófitos y criptófitos disminuyen (Tabla I).

La ordenación manual tradicional de la tabla inicial, utilizando especies diferenciales, entregó 7 grupos vegetacionales diferentes. Las especies diferenciales utilizadas fueron: Acaena integerrima, Aira praecox, Poa pratensis, Agrostis capillaris, Trifolium repens, Lolium perenne, Discaria chacaye y Nothofagus antarctica, de ellas, sólo la primera (subarbusto) y las dos últimas (arbustos) son plantas nativas, el resto corresponde a malezas herbáceas introducidas (Tabla II). Estas 8 especies diferenciales permitieron ordenar la tabla en 7 comunidades vegetales: matorral de ñire (Anemone multifida-Nothofagus antarctica), silvopastoreo con ñire, silvopastoreo con chacay, pradera fresca de trébolballica (Bromus catharticus-Trifolium repens), pradera de chépica-cadillo (Acaena ovalifolia-Agrostis capillaris) (cadillo = Acaena ovalifolia), pradera de cadillo-Aira (cadillo = Acaena integerrima) y estepa de cadillo (cadillo = Acaena integerrima), para las cuales en la Tabla III se indica grupo vegetacional (Gr), número de censos (Cs) y número de especies ( $\mathrm{Sp}$ ) por grupo, heterogeneidad del grupo (BC) nombre común y nombre científico de la comunidad vegetal, cuando ella ha sido descrita como asociación vegetal de acuerdo a las normas de nomenclatura fitosociológica vigentes.

El número de censos por comunidad resultó ser bastante variable, lo que de alguna manera está representando la abundancia (presencia de rodales) de cada una en el lugar de trabajo, siendo las praderas y estepa las mejor representadas. El número de especies fue mayor en el matorral de ñire y en la pradera de chépica-cadillo, le siguen la pradera de trébol-ballica, el silvopastoreo en chacay y la estepa de cadillo. El menor número de especies se presentó en el silvopastoreo con ñire y en la pradera de cadillo-Aira. La heterogeneidad del matorral de ñire, pradera de trébol-ballica y pradera de chépica-cadillo fue alta, mientras que en la pradera de cadillo-Aira y la estepa de cadillo ella fue menor, indicando uniformidad de los rodales. Los dos manejos de silvopastoreo (con ñire y con chacay) presentaron valores intermedios (Tabla III).

El análisis de conglomerados confirma la mayor homogeneidad del silvopastoreo en ñire, de la pradera de cadillo-Aira y de la estepa de cadillo con una distancia de conglomeración sobre $80 \%$ (Fig. 3 b, f, g), en cambio la pradera de chépica-cadillo, el silvopastoreo en chacay, la pradera de trébol-ballica y el matorral de ñire se presentan como muy heterogéneos, conglomerándose en distancias bajo $50 \%$ (Fig. 3 a, c, d, e) La pradera de chépica-cadillo (grupo E) muestra dos subgrupos no separables con la metodología tradicional, que se diferencian por la ausencia de Taraxacum officinale en el menor de ellos. La pradera de cadillo-Aira (grupo F) y la estepa de cadillo (grupo G) se unen a una distancia de alrededor de $70 \%$, demostrando relación de origen entre ambas (Fig. 3 número 1'). Estas a su vez se unen aunque con una mayor distancia al conglomerado que forman la pradera de trébol-ballica y el silvopastoreo en ñire, demostrando con ello también una relación de origen (Fig. 3 número 2'). Estos dos últimos conglomerados no están claramente delimitados lo que señala una gran proximidad entre ellos, incluso con censos de posición intermedia. Este análisis relaciona en su origen a estas 4 comunidades indicando que el silvopastoreo en ñire da origen a la pradera de trébol-ballica y ésta a su vez a la pradera de cadillo-Aira y a la estepa de cadillo. A los 4 conglomerados descritos se une el de la pradera de chépica-cadillo (grupo E), demostrando posiblemente un rezago de pastoreo, permitiendo el libre desarrollo de Agrostis capillaris (chépica) y de Acaena ovalifolia (cadillo). La segregación en dos subgrupos de esta pradera podría deberse a una diferente duración de este rezago. La unión de los grupos B, D, F y G al matorral de ñire (grupo A) podría indicar que este matorral constituye el origen de la degradación antrópica que pasaría desde un matorral, mediante silvopastoreo, a praderas y por último a una estepa. La posición muy aislada del silvopastoreo en chacay lo retira de la serie de degradación antrópica comentada (grupo C).

El análisis de ordenación, cuyos primeros dos ejes constituyen un 46,1\% de la varianza total, permite avanzar en la determinación de las relaciones de origen entre las comunidades que conforman el mosaico de vegetación estudiado. La Fig. 4 confirma que los grupos $\mathrm{F}$ y $\mathrm{G}$ (pradera de cadillo-Aira y estepa de cadillo) se encuentran florísticamente muy cerca entre sí y por lo tanto, uno pudo dar origen al otro. Lo mismo sucede con la pradera de trébol-ballica y con el silvopastoreo en ñire que también 
muestran una gran proximidad entre sí. El matorral de ñire (grupo A) da origen al silvopastoreo en ñire en tanto que la pradera de trébol-ballica sería el siguiente estadio en la sucesión secundaria de degradación. Nuevamente los grupos C (silvopastoreo en ñire) y E (pradera de chépica-cadillo) se presentan aislados sin sugerir relación de origen directa.

TABLA II. Especies diferenciales y acompañantes de las comunidades de vegetación estudiadas en Aisén. Abundancia de las especies en las comunidades calculadas como valor de importancia. Especies presentes en una sola comunidad (42) fueron excluidas. Para el significado de las letras que diferencian las comunidades ver Tabla III. Fr $=$ frecuencia.

TABLE II. Differential and associate species of the different communities studied in Aisen. Species abundance calculates as importance values. Species of one community (42) were excluded. For the meaning of the capital letters that differentiated the communities see Table III. $\mathrm{Fr}=$ frequency.

\begin{tabular}{|c|c|c|c|c|c|c|c|c|}
\hline COMUNIDADES VEGETALES: & A & B & $\mathrm{C}$ & $\mathrm{D}$ & $\mathrm{E}$ & $\mathrm{F}$ & G & $\mathrm{Fr}$ \\
\hline \multicolumn{9}{|l|}{ ESPECIES DIFERENCIALES } \\
\hline Acaena integerrima Gillies ex Hook. et Arn. & & & & & & 22 & 40 & 2 \\
\hline Aira praecox $\mathrm{L}$. & & & & & & 64 & 1 & 2 \\
\hline Poa pratensis L. & & & 5 & 4 & 2 & 7 & 1 & 5 \\
\hline Agrostis capillaris L. & & & 1 & 1 & 62 & & & 3 \\
\hline Trifolium repens $\mathrm{L}$. & & 20 & 4 & 22 & 4 & & & 4 \\
\hline Lolium perenne $\mathrm{L}$. & & 13 & 5 & 4 & & & & 3 \\
\hline Discaria chacaye (G. Don) Tortosa & 2 & 1 & 43 & & 1 & & & 4 \\
\hline Nothofagus antarctica (G. Forst.) Oerst. & 70 & 40 & 10 & 1 & 1 & 1 & 1 & 7 \\
\hline \multicolumn{9}{|l|}{ ESPECIES ACOMPAÑANTES } \\
\hline Taraxacum officinale Weber ex F.H. Wigg. & 1 & 60 & 43 & 39 & 10 & 10 & 1 & 7 \\
\hline Holcus lanatus $\mathrm{L}$. & 2 & 4 & 17 & 8 & 11 & 1 & 1 & 7 \\
\hline Cerastium arvense $\mathrm{L}$. & 1 & & 1 & 1 & 1 & 1 & 1 & 6 \\
\hline Anemone multifida Poir. & 15 & 1 & & 1 & 1 & 1 & 1 & 6 \\
\hline Achillea millefolium $\mathrm{L}$. & 1 & & & 5 & 14 & 1 & 4 & 5 \\
\hline Rumex acetosella $\mathrm{L}$. & 1 & & & 1 & 1 & 1 & 1 & 5 \\
\hline Acaena pinnatifida Ruiz et Pav. & 1 & & & 1 & 1 & 1 & 1 & 5 \\
\hline Hypochaeris radicata $\mathrm{L}$. & 1 & & 1 & 1 & 4 & & 1 & 5 \\
\hline Berberis microphylla G. Forst. & 1 & & 23 & 1 & 1 & & 1 & 5 \\
\hline Fragaria chiloensis (L.) Duch. & 2 & & 4 & 1 & 3 & & & 4 \\
\hline Carex fuscula D’Urv. & 1 & & 1 & 4 & 1 & & & 4 \\
\hline Centaurium littorale (Turner) Gilmour & 1 & & 1 & 1 & 1 & & & 4 \\
\hline Geranium berteroanum Colla & 1 & & 1 & 1 & & 1 & & 4 \\
\hline Crepis capillaris (L.) Wallr. & & & 1 & 1 & 2 & & & 3 \\
\hline Vulpia bromoides (L.) Gray & & & & 1 & & 1 & 1 & 3 \\
\hline Acaena ovalifolia Ruiz et Pav. & & 1 & & 1 & 1 & & & 3 \\
\hline Blechnum penna-marina (Poir.) Kuhn & 9 & 1 & 7 & & & & & 3 \\
\hline Osmorhiza berteroi DC. & 1 & 1 & & & & & 1 & 3 \\
\hline Geum magellanicum Pers. & & & 1 & 1 & 1 & & & 3 \\
\hline Quinchamalium chilense Molina & 1 & & 1 & & 1 & & & 3 \\
\hline Plantago lanceolata $\mathrm{L}$. & & & & & 1 & & 1 & 2 \\
\hline Aira caryophyllea $\mathrm{L}$. & & & & 6 & 1 & & & 2 \\
\hline Rosa rubiginosa $\mathrm{L}$. & & & & & 1 & & 1 & 2 \\
\hline Cirsium vulgare (Savi) Ten. & & & 2 & & 1 & & & 2 \\
\hline Prunella vulgaris $\mathrm{L}$. & & & 15 & & 1 & & & 2 \\
\hline Escallonia virgata (Ruiz et Pav.) Pers. & 1 & & & & 1 & & & 2 \\
\hline Plantago major $\mathrm{L}$. & & & 1 & & 1 & & & 2 \\
\hline
\end{tabular}




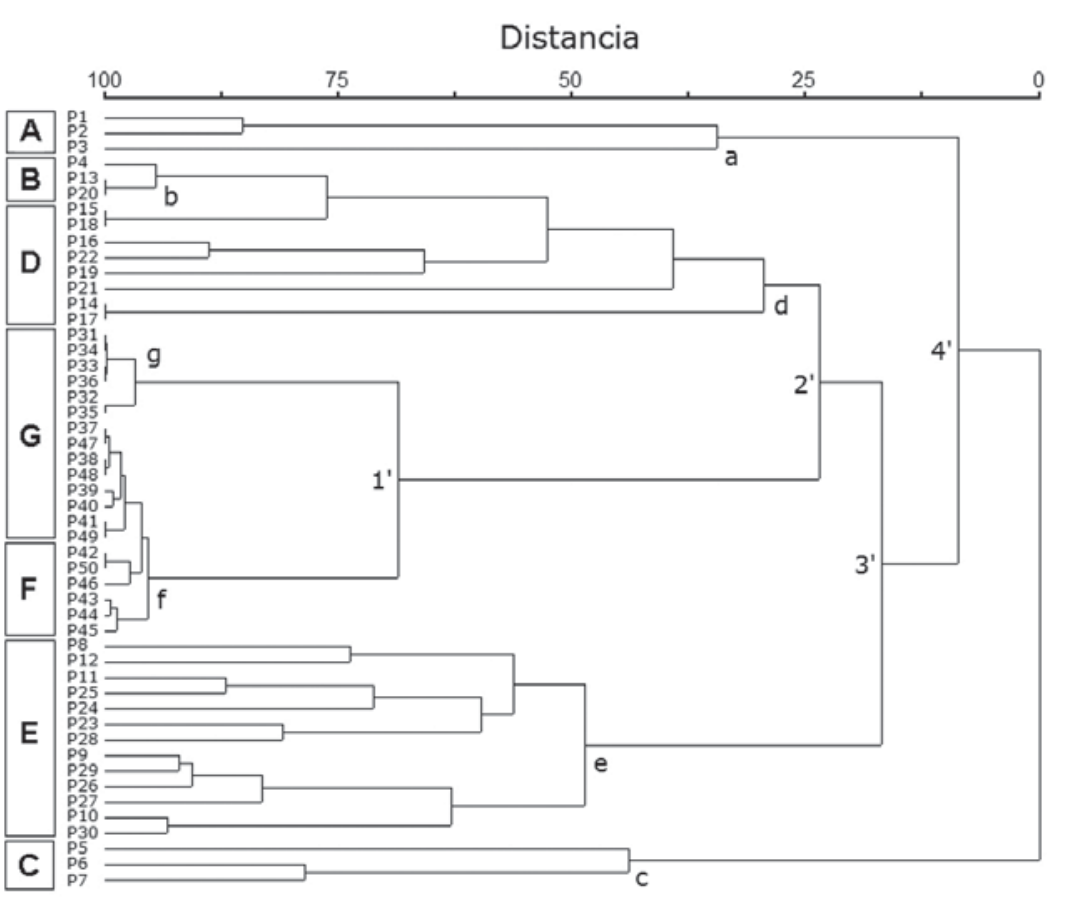

FIGURA 3. Dendrograma con la similitud florística (letras minúsculas) y posibles relaciones de origen (números prima) de los grupos de censos formados. Los números de la barra izquierda indican en orden correlativo las comunidades (Tabla III) de la A a la G. Explicaciones en el texto.

FIGURE 3. Dendrogram with the floristic similarity (small letter) and probably origin relationships (raw numbers) between the sample groups. The numbers of the left bars in correlative arrangement indicate the samples groups assimilate to communities (Table III) from A to G (Tabla III). Explanations in the text.

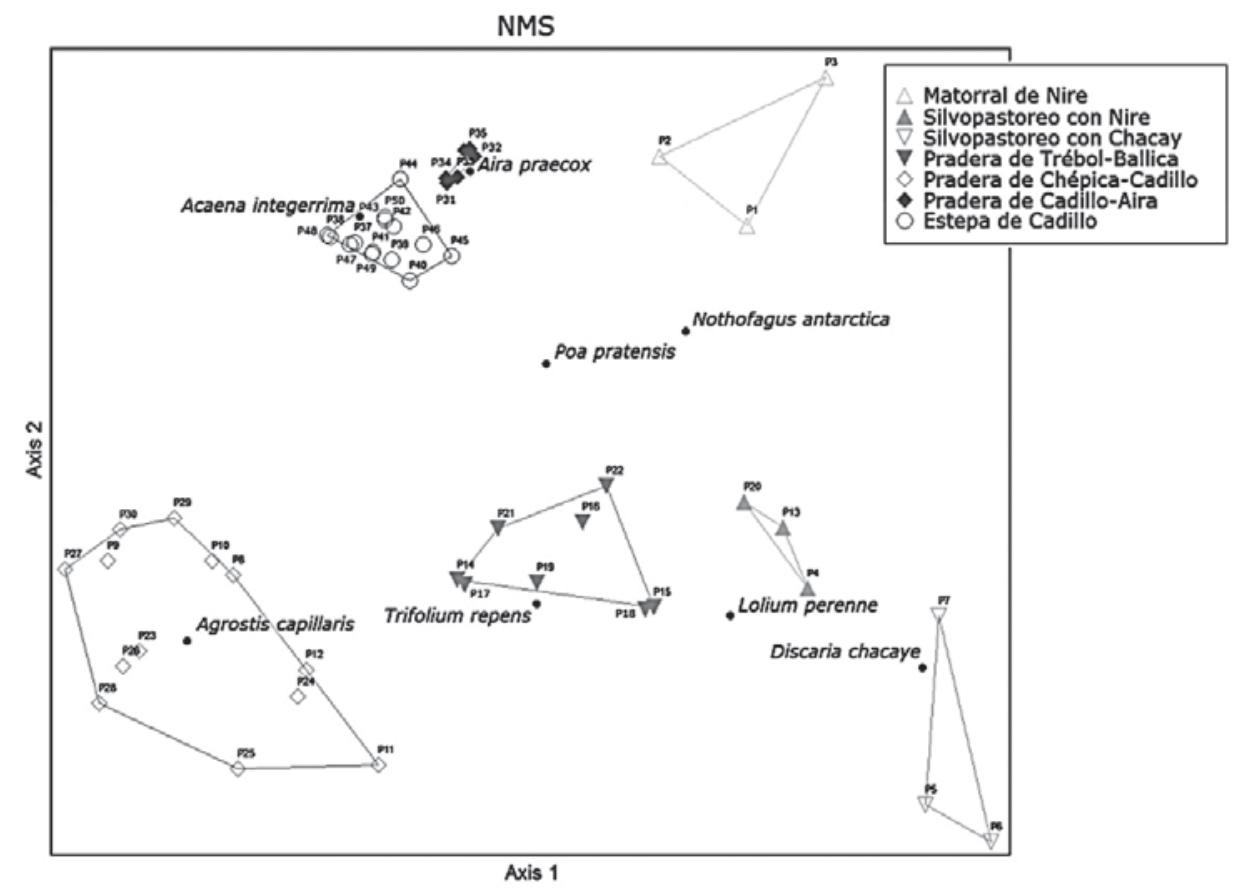

FiguRa 4. Distribución de los censos de vegetación en el plano formado por los dos primeros componentes principales. En la ordenación se indican las especies diferenciales de los grupos.

FIGURE 4. Distribution of the vegetation samples in the plane formed by the two first principal components. In the ordination is indicated the differential species of the groups. 
Tabla III. Comunidades vegetales estudiadas en Aisén. Gr: Grupo respectivo, Cs: esfuerzo de muestreo en censos, Sp: número total de especies, BC: heterogeneidad del grupo calculada como promedio del índice de Bray-Curtis entre los censos de una misma comunidad.

TABLE III. Plant communities studied in Aisen. Gr: Group, Cs: sample effort in samples, Sp: total number of species, BC: heterogeneity of each Group calculated as average of the Bray-Curtis index from the samples of each community.

\begin{tabular}{ccccll}
\hline Gr & Cs & Sp & BC & NomBRE COMÚN & COMUNIDAD \\
\hline A & 3 & 43 & 0,404 & matorral de ñire & $\begin{array}{l}\text { Anemone multifida-Nothofagus antarctica } \\
\text { (Vidal } \text { et al. 2011) }\end{array}$ \\
B & 3 & 11 & 0,258 & silvopastoreo con ñire & $\begin{array}{l}\text { Estado de degradación } \\
\text { Estado de degradación }\end{array}$ \\
C & 3 & 24 & 0,373 & silvopastoreo con chacay & $\begin{array}{l}\text { Bromus catharticus-Trifolium repens } \\
\text { (Oberdorfer 1960) }\end{array}$ \\
D & 8 & 29 & 0,518 & pradera de trébol-ballica & $\begin{array}{l}\text { Acaena ovalifolia-Agrostis capillaris } \\
\text { (Oberdorfer 1960) }\end{array}$ \\
E & 13 & 36 & 0,448 & pradera de chépica-cadillo & No descrita \\
F & 6 & 13 & 0,097 & pradera de cadillo-Aira & No descrita \\
\hline
\end{tabular}

TABLA IV. Similitud (triángulo superior) y disimilitud florística (triángulo inferior) entre los grupos vegetacionales, aplicando el coeficiente de comunidad de Ellenberg a la Tabla II. Letras mayúsculas indican los grupos o comunidades, cuyos nombres se pueden ver en la Tabla III.

TABLE IV. Similarity (above triangle) and disimilarity (below triangle) between the vegetation groups (Table II) with the Ellenberg community coefficient. The capital letters indicated the groups or community, listed in the Table III.

\begin{tabular}{cccccccc}
\hline Grupos & A & B & C & D & E & F & G \\
\hline A & - & 56,52 & 62,44 & 47,01 & 3669 & 27,54 & 35,97 \\
B & 43,48 & - & 67,34 & 72,39 & 28,64 & 30,10 & 37,37 \\
C & 37,56 & 32,66 & - & 53,30 & 75,00 & 19,37 & 27,09 \\
D & 52,99 & 27,61 & 46,70 & - & 78,65 & 24,52 & 29,81 \\
E & 63,31 & 71,31 & 25,00 & 21,35 & - & 15,00 & 19,17 \\
F & 72,46 & 69,90 & 80,63 & 75,48 & 85,00 & - & 91,16 \\
G & 64,03 & 62,63 & 72,91 & 70,19 & 80,83 & 8,84 & - \\
\hline
\end{tabular}

Los mismos grupos de vegetación fueron ordenados en una constelación de comunidades confeccionadas con las matrices de similitud y disimilitud de la Tabla IV. Este arreglo muestra que los grupos $\mathrm{F}$ (pradera de cadillo-Aira) y $\mathrm{G}$ (estepa de cadillo) tienen alta afinidad entre ellos, pero se sitúan muy alejados del resto ocupando uno de los vértices de la constelación (Fig. 5), señalando con esto, seguramente, un estadio avanzado en la degradación de la vegetación. También se aprecia la gran afinidad florística existente entre los grupos B (silvopastoreo con ñire) y D (pradera fresca de trébol-ballica), lo que señala una relación de origen entre ambos. Ellos se encuentran más o menos equidistantes de los grupos A (matorral de ñire), C (silvopastoreo con chacay) y E (pradera de chépica-cadillo), más separados del resto.

En el espectro biológico los grupos A y B presentan la mayor cantidad de fanerófitos, lo que indica una mayor proximidad a la vegetación primaria constituida por formaciones leñosas, naturalmente esto podría corresponder también a los primeros estadios de degradación o una recuperación del elemento leñoso nativo en una posible regeneración en curso de la vegetación primaria (Fig. 6). Los caméfitos, que aparecen en condiciones extremas, 
están escasamente representados en todos los grupos; por el contrario, los hemicriptófitos (hierbas perennes) tienen alta representatividad en todos ellos, lo que se corresponde con el macroclima de la región y con la ganadería como principal manejo del suelo. Los terófitos (hierbas anuales) están mejor representados en las comunidades pratenses, indicando un distanciamiento de la vegetación primaria primitiva y un aumento del xerofitismo.

Al analizar la relación entre las especies nativas e introducidas de cada grupo se observa que en A (matorral de ñire) y B (silvopastoreo con ñire) dominan especies nativas, mientras que en $\mathrm{E}$ (pradera de chépica-cadillo), $\mathrm{F}$ (pradera de cadillo-Aira) y G (estepa de cadillo) lo hacen las especies introducidas (Fig. 6, gráfico inferior derecho). Los grupos C (silvopastoreo con chacay) y D (pradera fresca de trébol-ballica) presentan valores más o menos equilibrados de ambos contingentes. Lo anterior coloca en el origen de la degradación antropogénica del área de estudio al matorral

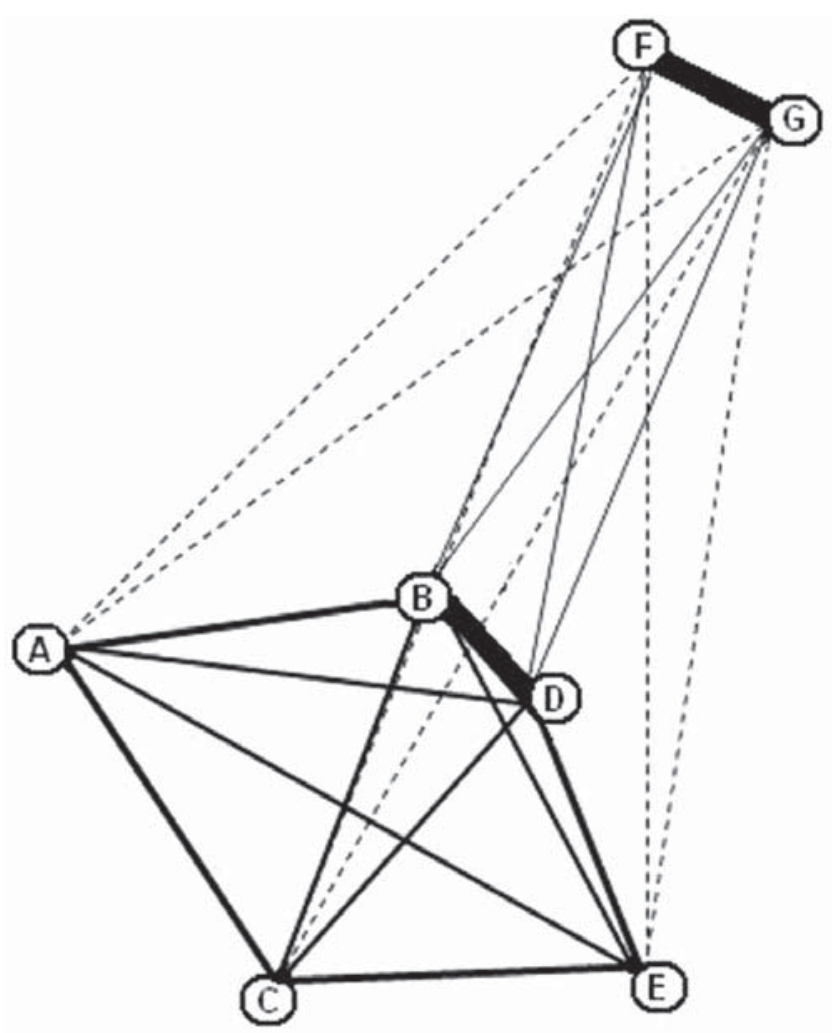

Figura 5. Constelación de las comunidades diferenciadas. A: matorral de ñire, B: silvopastoreo con ñire, C: silvopastoreo con chacay, D: pradera de trébol-ballica, E: pradera de chépica-cadillo, F: pradera de cadillo-aira, G: estepa de cadillo. Las líneas más gruesas indican mayor similitud florística.

FIGURE 5. Constellation of the differentiated communities. A: ñire scrub, B: ñire silvo-pasture, C: chacay silvo-pasture, D: trébolballica prairie, E: chépica-cadillo prairie, F: cadillo-aira prairie, G: cadillo steppe. The thiker lines indicated more floristic similarity. de ñire y al silvopastoreo en ñire, mientras que las otras comunidades representan estadios avanzados de ella.

Con los resultados descritos se propone que la posible posición espacial y temporal en la sucesión secundaria de degradación del matorral de ñire seguiría la siguiente dirección: la introducción del ganado en primera instancia degrada el matorral de ñire a un silvopastoreo con ñire cuya degradación finaliza en la instalación de una pradera fresca de trébol-ballica, la que por sobrepastoreo degrada a una pradera seca de cadillo-Aira, que por último se transforma en una estepa de cadillo, en ambos casos se trata de Acaena integerrima un subarbusto nativo.

\section{DISCUSIÓN}

Según los resultados y el análisis anterior, se puede plantear que el proceso de transformación del paisaje se inicia con la destrucción o alteración de la vegetación primitiva por diferentes causas (fuego, tala, raleo, floreo, "limpieza del bosque"), donde la posterior introducción de ganado crea formaciones pratenses mantenidas por el pastoreo, éstas por sobrepastoreo se degradan a distintos estadios que al ser abandonados son cubiertos generalmente por leñosas alóctonas, formándose nuevas comunidades, la mayoría de ellas arbustivas (Ramírez et al. 1988). En los estadios abandonados no se puede recuperar espontáneamente la vegetación original, tanto por la alteración física y química del suelo como también por la presencia de plantas leñosas invasoras alóctonas, que desplazan la flora nativa (Ellies et al. 1994).

Por la posición incierta de los grupos C (silvopastoreo en chacay) y E (pradera de chépica-cadillo) suponemos que el primero es una comunidad de lugares húmedos, ubicada en depresiones y resultante de la degradación del bosque de lenga, por lo tanto también evolucionaría hacia una pradera (húmeda) que no fue analizada (Vidal et al. 2011). Como se ha observado en otros lugares, la pradera de chépicacadillo podría resultar del retiro del pastoreo de la pradera de trébol-ballica (Ramírez et al. 1997a).

De acuerdo a lo constatado en terreno es posible que en algunos sectores el matorral de ñire (que tiene muchas especies alóctonas) podría tener origen en la degradación del primitivo bosque de lenga. De la misma manera, la estepa de cadillo podría evolucionar a una estepa de Mulinum spinosum (neneo), que en el área de estudio también es secundaria; mientras que en la zona esteparia oriental de Aisén, es primaria (Pisano 1981). Lo anterior podría modificar la dinámica vegetacional ya descrita del lugar estudiado como lo indica las Figuras 7 y 8.

Aunque el origen de la flora sigue siendo un importante indicador del estado de alteración de una comunidad vegetal, al agregar las formas de vida del espectro biológico se obtiene una mayor aproximación, especialmente para 
identificar el factor que ha provocado el cambio antrópico y el nivel de degradación alcanzado. En el caso estudiado el alto número de especies alóctonas indica un fuerte estado de antropización en la vegetación, pero el análisis de las formas de vida permite establecer las causas del cambio a la introducción y permanencia de ganado (bovino primero y ovino, después) que transporta los propágulos de especies resistentes al pastoreo y al pisoteo (Alvarez 2008). Además, se presentan formas indicadoras de degradación de la vegetación y del suelo, que provocarían xerofitismo, haciendo desaparecer parte de las especies introducidas, especialmente aquellas perennes en favor de aquellas anuales (terófitos) con un corto ciclo de vida (Hauenstein et al. 1988)
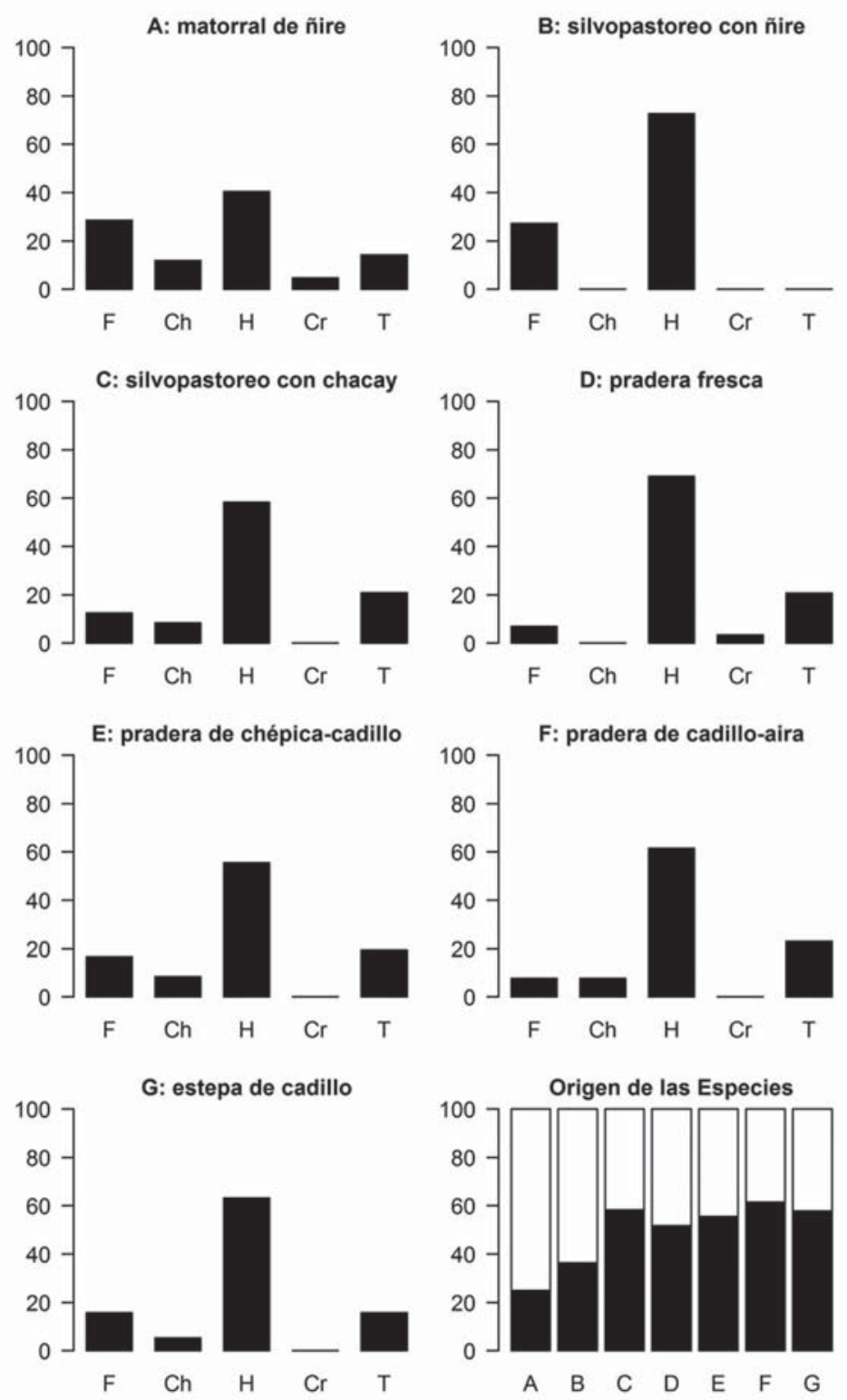

FIgURA 6. Espectros biológicos de las comunidades vegetales estudiadas en Aisén considerando la proporción de especies por forma de vida. F: fanerófitos, Ch: caméfitos, H: hemicriptófitos, Cr: criptófitos, T: terófitos. Gráfico inferior derecho muestra proporción de especies nativas (barras blancas) y especies introducidas (barras negras) en cada comunidad, cuyas abreviaturas del eje horizontal están explicadas en la Tabla III.

FIGURE 6. Biological spectrum of the plant communities studied in Aisen, considered the life forms of the species: F: phanerophytes, Ch: chamaephytes, H: hemicriptophyte, Cr: criptophyte, T: Therophytes. Right inferior graphic showing the number of native species (white barrs) and foreign species (black barrs) into each community. The abreviations of the communities in the horizontal axis are noted in the Table III. 


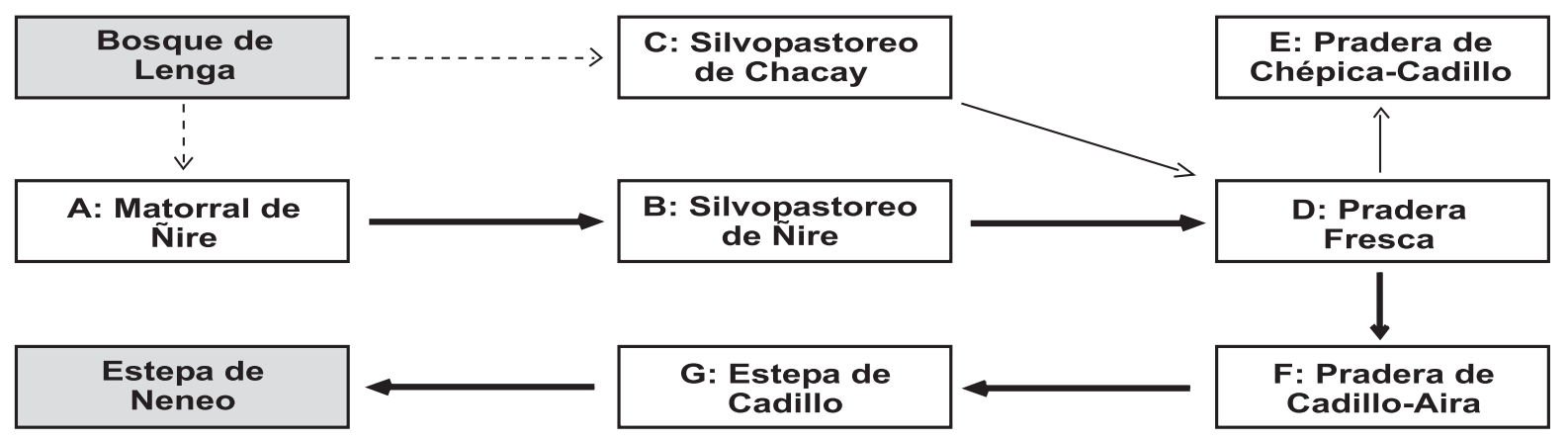

FiguRa 7. Dinámica de vegetación propuesta para las comunidades estudiadas. Las flechas más gruesas indican relaciones sustentadas por los análisis estadísticos y la constelación de la Figura 6, las delgadas indican propuestas con menos seguridad y las cortadas, relaciones probables con comunidades no estudiadas en este trabajo (cuadros grises).

FIGURE 7. Proposed vegetation dynamic for the studied communities. The thick arrows indicated the relationships suggested by the statistical multivariate analysis and the communities constellation of the Figure 6, the thin arrows indicated less security, and the broken arrows showing the not studied communities (gray boxes).

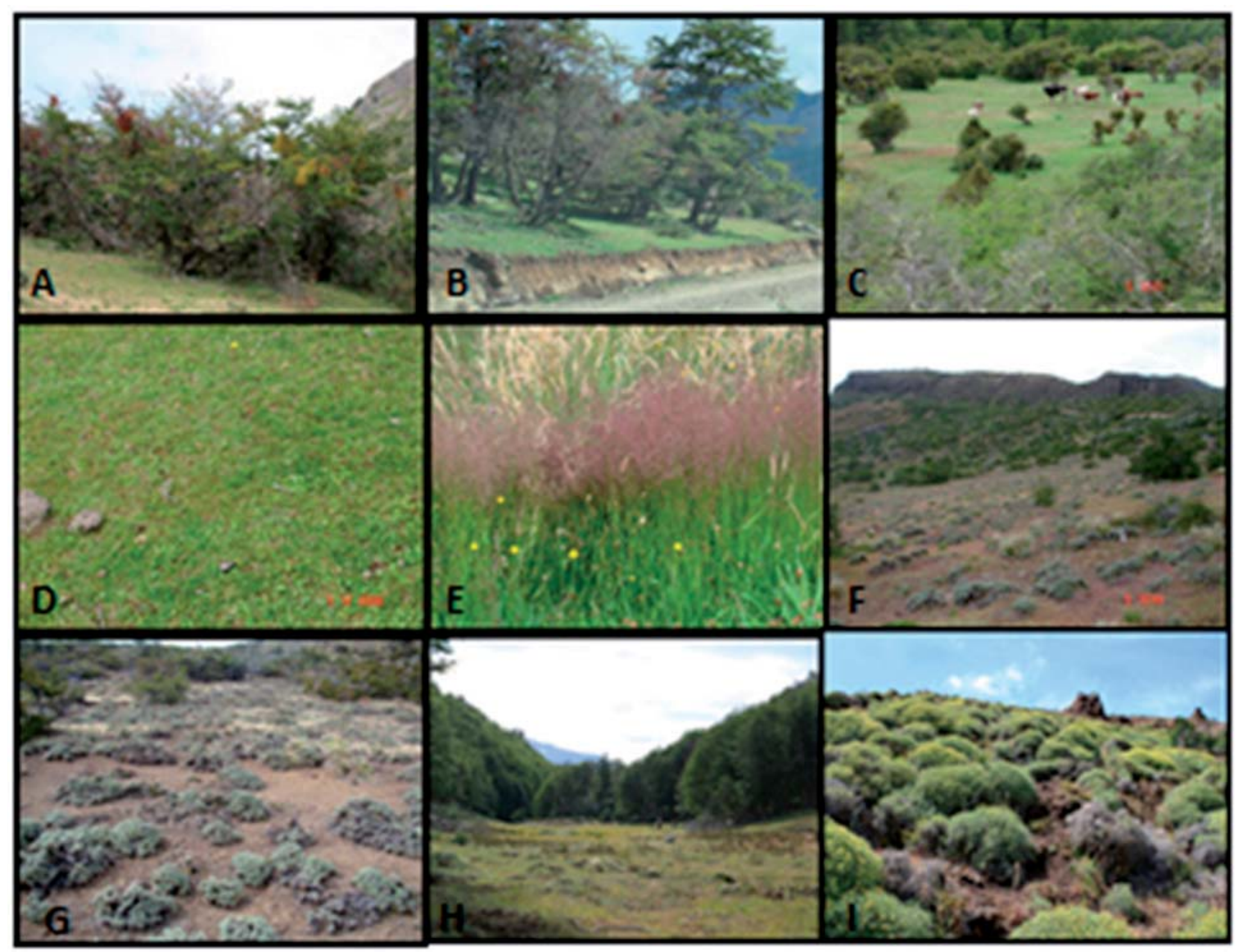

Figura 8. Comunidades vegetales trabajadas y mencionadas. $\mathrm{A}=$ matorral de ñire, $\mathrm{B}=$ silvopastoreo con ñire, $\mathrm{C}=$ silvopastoreo con chacay, $\mathrm{D}=$ pradera de trébol-ballica, $\mathrm{E}=$ pradera de chépica-cadillo, $\mathrm{F}=$ pradera de cadillo-aira, $\mathrm{G}=$ estepa de cadillo, $\mathrm{H}=$ bosque de lenga , I = estepa de neneo.

Figure 8. Plant communities worked and mentioned. $\mathrm{A}=$ ñire scrub, $\mathrm{B}=$ ñire silvo-pasture, $\mathrm{C}=$ chacay silvo-pasture, $\mathrm{D}=$ trébol-ballica prairie, $\mathrm{E}$ = chépica-cadillo prairie, $\mathrm{F}$ = cadillo-aira prairie, $\mathrm{G}$ = cadillo steppe, $\mathrm{H}=$ lenga forest, $\mathrm{I}=$ neneo steppe. 
El análisis de conglomerados permitió relacionar las distintas comunidades y establecer la distancia de su separación, lo que facilita la diferenciación de aquellas comunidades que están relacionadas en su origen. El análisis de ordenación permitió inferir el curso seguido por la degradación, es decir, por la transformación de las comunidades. Si estos resultados se complementan con la constelación y con aquéllos de presencia de especies alóctonas en cada comunidad, es posible determinar la posición de la comunidad en la dinámica de vegetación, es decir, su carácter original primario o secundario antropogénico. Lo anterior, permite confirmar también que un número importante de comunidades vegetales puede tener dos orígenes, uno original y otro secundario o terciario, como sucede con la estepa de neneo (Pisano 1985), con la cual terminaría la serie de degradación estudiada estabilizándose en una situación que, aunque improductiva, protege el suelo degradado y lo recupera, debido a la presencia en la estepa de leguminosas fijadoras de nitrógeno. Sin embargo, esta comunidad terminal es primaria en el clima estepario de la Patagonia. En este estadio terminal dominan los caméfitos pulviniformes, forma de vida esteparia que soporta condiciones extremas.

Independiente de las quemas (incendios voluntarios) realizados en la región en el siglo pasado (Le Quesne 2002, Guerra 2002), hoy en día los rodales de bosque son raleados para obtener combustible doméstico y material de construcción, esto se realiza en forma aleatoria, sin un plan preconcebido, sino dependiendo más bien de la disponibilidad y de la accesibilidad al recurso (Veblen et al. 1995). Posteriormente, se procede a "limpiar el bosque" ya raleado, es decir, a retirar el sotobosque transformándolo en una especie de parque en el cual se introduce el ganado, este agente degradante cambia la composición florística del estrato herbáceo, al alterar el balance de nutrientes con sus excrementos y la porosidad del suelo con el pisoteo, situación que es aprovechada por muchas malezas que pueden desarrollarse debido a la naturaleza caducifolia del bosque que permite la entrada de luz en primavera. Este proceso es aumentado con la disminución de tamaño de los rodales boscosos o arbustivos. En un estado avanzado desaparecen los árboles por muerte o por tala y principalmente por falta de regeneración, siendo reemplazados por matorrales ralos, que funcionan como un silvopastoreo y que posteriormente, se transforman en praderas antropogénicas permanentes, las que al degradarse por el sobrepastoreo son ocupadas por estepas secundarias (León \& Aguilar 1985). Sin embargo, en lugares más favorables, las praderas abandonadas pueden ser invadidas por malezas leñosas arbustivas que forman las llamadas "asociaciones permanentes", pobres en especies e improductivas para el hombre (Ramírez et al. 1988). En la región de trabajo cumple un rol muy importante en este proceso la maleza alóctona rosa mosqueta (Rosa rubiginosa) de origen europeo, diseminada por caballares.
En condiciones de mayor humedad, el mismo rol lo cumple el arbusto nativo llamado "chapel" (Escallonia virgata) que forma densos matorrales, tupidos e impenetrables, donde no es posible aplicar otros manejos.

\section{AGRADECIMIENTOS}

Esta investigación fue realizada en el marco del Proyecto CA 012.05 UACh-HIDROAYSEN. Nuestros agradecimientos a Johana Villagra que ayudó en el levantamiento de censos. Las excursiones de M. Alvarez fueron apoyadas por la A.F.W. Schimper-Stiftung. C. Novoa agradece la ayuda de CONICYT y DAAD por la beca que le permite realizar estudios de doctorado en Alemania y al Prof. Dr. R. Pott por la acogida en su instituto.

\section{BIBLIOGRAFÍA}

Aguayo, M., A. Pauchard, G. Azócar \& O. Parra. 2009. Cambio de uso del suelo en el centro-sur de Chile a fines del siglo XX. Entendiendo la dinámica espacial y temporal del paisaje. Revista Chilena de Historia Natural 82: 361-374.

Álvarez, M. 2008. Diasporenbank und Ökologie der Vegetation temporärer Gewässer im Cholchol-Gebiet (9. Region, Chile) Dissertationes Botanicae 407: 1-87.

Amigo, J. \& C. Ramírez. 1998. A bioclimatic classification of Chile woodland communities in the temperate zone. Plant Ecology 136: 9-26.

Amigo, J. \& M. Rodríguez-Guitián. 2011. Bioclimatic and phytosociological diagnosis of the species of the Nothofagus genus (Nothofagaceae) in South America. International Journal of Geobotanik Research 1: 1-20.

Braun-Blanquet, J. 1979. Fitosociología, bases para el estudio de las comunidades vegetales. H. Blume Ediciones, Madrid. $820 \mathrm{pp}$.

Bray, J.R. \& J.T. Curtis. 1957. An ordination of the upland forest communities of southern Wisconsin. Ecological Monographs. 27: 325-349.

CaIn, S. 1950. Life forms and phytoclimate. Botanical Review 16: $1-31$.

De Vries, D.M., J.P. Baretta \& G. Hamming. 1954. Constellation of frequent herbage plants, based on their correlation in ocurrence. Vegetatio 5-6: 105-111.

DierschKe, H. 1994. Pflanzensoziologie. Eugen Ulmer, Stuttgart. 683 pp.

Ellies, A. \& C. Ramírez. 1994. Efecto del manejo sobre la estructura del suelo y la biodiversidad específica vegetal. Seminario Medio Ambiente, Biodiversidad y Actividades Productivas, Santiago 4: 79-103.

Ellies, A., R. Grez \& C. Ramírez. 1994. Efecto del manejo sobre la estructura del suelo. Serie Carillanca 43: 13-29.

Frey, W. \& R. LöSch. 2010. Geobotanik - Pflanze und Vegetation in Raum und Zeit. Spektrum Akademischer Verlag, Heidelberg. 600 pp.

Guerra, G. 2002. El fuego: sus daños y efectos. En: A. Baldini \& L. Pancel (eds.) Agentes de daño en el bosque nativo. pp. 
52-45. Editorial Universitaria, Santiago, Chile.

HaJek, E. \& F. Di CAstri. 1975. Bioclimatografía de Chile. Editorial Universidad Católica de Chile, Santiago. 107 pp.

Hauenstein, E., C. Ramírez, M. Latsague \& D. Contreras. 1988. Origen fitogeográfico y espectro biológico como medida del grado de intervención antrópica en comunidades vegetales. Medio Ambiente 9(1): 140-142.

IzCo, J. \& M. DEL ARCO. 2003. Código internacional de nomenclatura fitosociológica. Materiales didácticos universitarios, Serie Botánica 2. Universidad de La Laguna, Santa Cruz de Tenerife. $155 \mathrm{pp}$.

KnAPP, R. (ed.) 1984. Sampling methods and taxon analysis in vegetation sciences. Dr. W. Junk Publishers, La Haya, Boston, Lancaster. $370 \mathrm{pp}$.

KreEb, K. 1983. Vegetationskunde. UTB Grosse Reihe, Ulmer, Stuttgart. $331 \mathrm{pp}$.

Leon, R.J.C. \& M.R. Aguilar. 1985. El deterioro por uso pasturil en estepas herbáceas patagónicas. Phytocoenologia 13(2): $181-196$

Le Quesne, C. 2002. Bioecología del bosque nativo chileno. En: A. Baldini \& L. Pancel (eds.) Agentes de daño en el bosque nativo. pp. 19-44. Editorial Universitaria, Santiago, Chile.

LeYer, I. \& K. Wesche. 2007. Multivariate Statistik in der Ökologie. Springer, Berlin. $221 \mathrm{pp}$.

Luebert, F. \& P. Pliscoff. 2006. Sinopsis bioclimática y vegetacional de Chile. Editorial Universitaria, Santiago. $316 \mathrm{pp}$.

Matthei, O. 1995. Manual de las Malezas que crecen en Chile. Alfabeta Impresores, Santiago. Chile. 545 pp.

Mc Cune, B. \& G. JAMES. 2002. Analysis of ecological communities. MjM Sofware, Gleneden Beach, Oregon, USA. 304 pp.

Moore, D.M. 1983. Flora of Tierra del Fuego. Anthony Nelson Publishing, Shropshire, \& Missouri Botanical Garden, St. Louis. 396 pp.

Mueller-Dombois, D. \& H. Ellenberg. 1974. Aims and Metods of the vegetation science. John Wiley \& Sons, New York. $302 \mathrm{pp}$.

Oberdorfer, E. 1960. Pflanzensoziologischen Studien in Chile, Ein Vergleich mit Europa. Flora et Vegetatio Mundi 2: 1108.

Pisano, E. 1981. Bosquejo fitogeográfico de Fuego-Patagonia. Anales Instituto. Patagonia. 12: 159-171.

PisAno, E. 1985. La estepa patagónica como recurso pastoril en Aysén y Magallanes. Ambiente y desarrollo 1(2): 45-59.

Ramírez, C., J. Amigo \& C. SAn Martín. 2003. Vegetación pratense litoral y dinámica vegetacional antropogénica en Valdivia, Chile. Agro Sur 31(1): 24-37.
Ramírez, C., J. Barrera, D. Contreras \& J. San Martín. 1988. Estructura y regeneración del matorral de Ulex europaeus en Valdivia, Chile. Medio Ambiente 9(1): 143-149.

Ramírez, C., M. Moraga \& H. Figueroa. 1984. La similitud florística como medida de degradación antrópica del bosque valdiviano. Agro Sur 12(2): 127-139.

Ramírez, C., C. SAn Martín \& P. OJeda. 1997. Muestreo y tabulación fitosociológica aplicados al estudio de los bosques nativos. Bosque 18(2): 19-27.

Ramírez, C., C. San Martín, A. Ellies \& R. Mac Donald. 1997a. Cambios florísticos, fitosociológicos y edáficos provocados por exclusión de pastoreo en una pradera valdiviana, Chile. Agro Sur 24(2): 180-195.

Reque, J.A., M. Sarasola, J. Gyenge \& M.E. Fernández. 2007. Caracterización silvícola de ñirantales del norte de la Patagonia para la gestión forestal sostenible. Bosque 28(1): 33-45.

Rodríguez, R., A. Marticorena \& E. Teneb. 2008. Plantas vasculares de los ríos Baker y Pascua, Región de Aisén, Chile. Gayana Botánica 65: 39-70.

Rosas, C. 1991. Estudio de la vegetación leñosa en áreas afectadas por deslizamientos en el parque Nacional Puyehue, Osorno, Décima Región. Tesis, Facultad Ciencias Forestales, Universidad Austral de Chile, Valdivia, Chile. 69 pp.

San Martín, C., J. Villagra \& C. Novoa. 2009. Comparación de manejos pratenses del centro-sur de Chile utilizando valores bioindicadores de Ellenberg. Gayana Botánica 66(2): $158-170$

Terradas, J. 2006. Ecología de la vegetación - De la ecología de las plantas a la dinámica de comunidades y paisajes. Ediciones Omega, Barcelona. 703 pp.

Veblen, T., T. Kitzberger, B. Burns \& A. Rebertus. 1995. Perturbaciones y dinámica de regeneración en bosques andinos del Sur de Chile y Argentina. En: J. Armesto, C. Villagrán \& M.T.K. Arroyo (eds.) Ecología de los bosques nativos chilenos. pp. 169-213. Editorial Universitaria, Santiago, Chile.

Vidal, O., J.R. Bannister, V. Sandoval, Y. Pérez \& C. Ramírez. 2011. Woodland communities in the Chilean cold-temperate zone (Baker and Pascua basins): Floristic composition and morpho-ecological transition. Gayana Botanica 68(2): 141-154.

Wikum, D. \& G.F. Shanholtzer. 1978. Application of the BraunBlanquet cover-abundance scale for vegetation analysis in land development studies. Environmental Management 2(4): 323-329.

Recibido: 18.04.12

Aceptado: 17.07 .12 